\title{
Infection against infection: parasite antagonism against parasites, viruses and bacteria
}

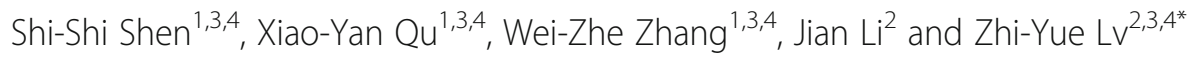

\begin{abstract}
Background: Infectious diseases encompass a large spectrum of diseases that threaten human health, and coinfection is of particular importance because pathogen species can interact within the host. Currently, the antagonistic relationship between different pathogens during concurrent coinfections is defined as one in which one pathogen either manages to inhibit the invasion, development and reproduction of the other pathogen or biologically modulates the vector density. In this review, we provide an overview of the phenomenon and mechanisms of antagonism of coinfecting pathogens involving parasites.

Main body: This review summarizes the antagonistic interaction between parasites and parasites, parasites and viruses, and parasites and bacteria. At present, relatively clear mechanisms explaining polyparasitism include apparent competition, exploitation competition, interference competition, biological control of intermediate hosts or vectors and suppressive effect on transmission. In particular, immunomodulation, including the suppression of dendritic cell (DC) responses, activation of basophils and mononuclear macrophages and adjuvant effects of the complement system, is described in detail.
\end{abstract}

Conclusions: In this review, we summarize antagonistic concurrent infections involving parasites and provide a functional framework for in-depth studies of the underlying mechanisms of coinfection with different microorganisms, which will hasten the development of promising antimicrobial alternatives, such as novel antibacterial vaccines or biological methods of controlling infectious diseases, thus relieving the overwhelming burden of ever-increasing antimicrobial resistance.

Keywords: Antagonism, Parasite, Coinfection, Pathogen, Immunomodulation

\section{Multilingual abstracts}

Please see Additional file 1 for translations of the abstract into the five official working languages of the United Nations.

\section{Background}

Infectious diseases are imposing a considerable socioeconomic burden on countries with a wide distribution of pathogens. According to statistics from the World Health Organization (WHO), in 2016, lower respiratory

\footnotetext{
* Correspondence: Ivzhiyue@mail.sysu.edu.cn

Shi-Shi Shen, Xiao-Yan Qu and Wei-Zhe Zhang are the joint first authors

${ }^{2}$ Fifth Affiliated Hospital, Zhongshan School of Medicine, Sun Yat-sen

University, ZhuHai, Guangdong, China

${ }^{3}$ Key Laboratory of Tropical Disease Control, Ministry of Education, Sun

Yat-sen University, Guangzhou, China

Full list of author information is available at the end of the article
}

infections ranked $3^{\text {rd }}$ among the top 10 causes of death, while diarrhoeal diseases caused by pathogenic infection ranked $8^{\text {th }}$, leading to an alarming death toll of millions of individuals worldwide [1]. More seriously, because of the rapid development of antimicrobial resistance (AMR) among pathogens, antibiotics are gradually losing their expected therapeutic capacity [2]. Therefore, the discovery of an alternative treatment strategy for controlling infections is imperative. Previous studies have revealed an interesting "infection against infection" relationship among various pathogens [3, 4]. In the antagonistic phenomenon, one pathogen from a certain species manages to suppress other species of pathogens during concurrent infections through either of the following two processes: biologically controlling pathogen vectors or inhibiting the invasion, development and reproduction of other pathogens. In this review,

(c) The Author(s). 2019 Open Access This article is distributed under the terms of the Creative Commons Attribution 4.0 International License (http://creativecommons.org/licenses/by/4.0/), which permits unrestricted use, distribution, and 
antagonistic relationships during coinfection that specifically involve parasites are summarized and discussed to motivate further research on the cusp of revealing or even utilizing the biological principles behind antagonism in tropical disease control. Taking Wolbachia as a quintessential example, this genus of gram-negative bacteria succeeds in limiting the transmission of parasites, such as malaria parasites and Filaria as well as emerging human arboviruses, when transferred into mosquitoes [5-11]. Additionally, certain nematodes and bacteria not only block the transmission of some trematodes by biologically controlling intermediate hosts but also relieve symptoms by suppressing infections by viruses and malaria parasites [12-14]. The "infection against infection" antagonism between parasites and parasites, parasites and viruses, or parasites and bacteria has been gradually applied to humans and has provided new insights into the development of novel effective antimicrobial therapies.

\section{Main text}

\section{Literature Searching Strategy}

Figure 1 presents the entire literature review process. In the present paper, we reviewed scientific studies published from 1970 to 2018 to identify studies focusing on antagonism among parasites and other pathogens during coinfection. A comprehensive search strategy was developed in PubMed, employing proper key words and free text terms. The search terms were "Coinfection" [Mesh] AND ("parasitology" [Subheading] OR "parasitology"[All Fields] OR "parasites" [All Fields] OR "parasites" [MeSH Terms]). Selected reference lists of retrieved articles were also searched manually using the online databases PubMed (https://www. ncbi.nlm.nih.gov/pubmed/) or Web of Science (http://apps. webofknowledge.com/UA_GeneralSearch_input.do?product $=$ UA\&search_mode $=$ GeneralSearch $\&$ SID $=5$ ChWtwlrip
dAqJRyqsq\&preferencesSaved=). In addition, data about the incidence of diseases and antimicrobial resistance were obtained from the WHO website (http://www.who.int/). All identified articles were screened. The inclusion criteria were (1) studies that were published from Jan 1970 to Oct 2018; (2) studies that were published in English; and (3) studies that focused on antagonism between parasites and other pathogens. The exclusion criteria included (1) studies whose full articles were not available online either for free or with subscription (payment); (2) non-English literature; (3) studies irrelevant to the antagonism between parasites and other pathogens; and (4) poor-quality studies.

Two reviewers independently screened the PubMed electronic database to identify potentially eligible articles based on their titles and abstracts. All the research articles that were identified from searches of the electronic database were imported into a reference management software (EndNote X9, Thomson Corporation, Stanford, USA). Then, the study quality was independently assessed by two skilled researchers. The reference lists of the related articles were also checked to avoid missing relevant studies. The full text of each article was assessed carefully for inclusion or exclusion in the study when the information in the title and abstract was inadequate. Finally, we reviewed the identified studies to evaluate the eligibility on the basis of the inclusion and exclusion criteria. All the above procedures were conducted by two independent and trained researchers at Zhongshan School of Medicine in Sun Yat-sen University, and inconsistency between authors was resolved through discussions.

\section{Phenomena of infection against infection}

Previous studies have shown the antagonistic interaction between parasites and parasites, parasites and viruses, and parasites and bacteria (Table 1). These "infection

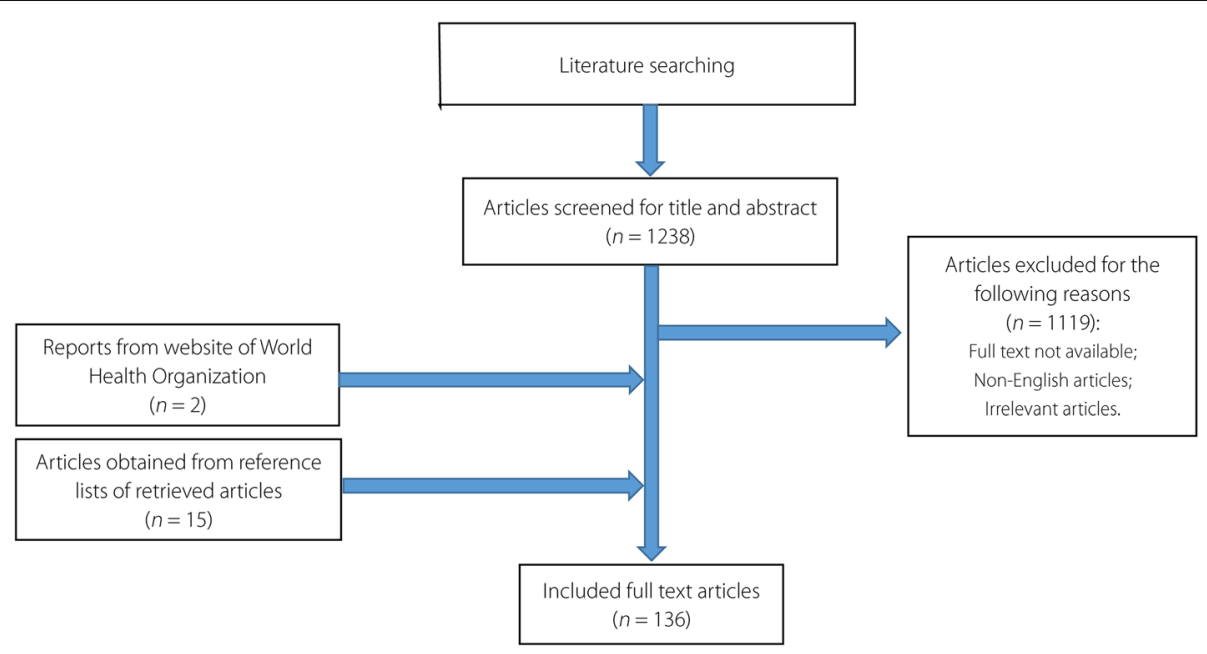

Fig. 1 Flow chart of selection process of the included and excluded articles 
Table 1 Phenomena of "infection against infection"

\begin{tabular}{|c|c|c|c|c|}
\hline Categories & Interspecies antagonism & Patterns of antagonism & $\begin{array}{l}\text { Intensity of } \\
\text { antagonism }\end{array}$ & References \\
\hline \multirow{13}{*}{$\begin{array}{l}\text { Between Parasites } \\
\text { and parasites }\end{array}$} & Heterorhabditis baujardi against Fasciola hepaticac; & Inhibiting transmission & $66.66 \%$ & [12] \\
\hline & H. baujardi against $F$. gigantica & Inhibiting transmission & $66.66 \%$ & [12] \\
\hline & Angiostrongylus cantonensis against Schistosoma mansonic; & Inhibiting transmission & $12.45 \%$ & [13] \\
\hline & Trichinella spiralis against Plasmodium bergheic; & Decreasing parasitaemia & $47.50 \%$ & [15] \\
\hline & Ascaris lumbricoides against $P$. falciparum ${ }^{\mathrm{a}, \mathrm{d}}$ & Attenuating symptoms & $24.00 \%$ & [16] \\
\hline & Trichuris trichiura against & Attenuating symptoms & $27.50 \%$ & {$[17]$} \\
\hline & P. falciparum ${ }^{d}$ & Decreasing parasitaemia & $28.00 \%$ & {$[18,19]$} \\
\hline & S. haematobium against $P$. falciparum ${ }^{a}$ & Attenuating symptoms & $76.00 \%$ & {$[20]$} \\
\hline & S. mansoni against $P$. knowlesi; & Inhibiting propagation & $88.82 \%$ & [21] \\
\hline & S. mansoni against $P$. yoelii ${ }^{a}$; & Inhibiting propagation & $31.70 \%$ & [22] \\
\hline & Litomosoides sigmodontis against $P$. berghei; & Inhibiting propagation & $22.00 \%$ & [23] \\
\hline & Trichostrongylus colubriformis against Haemonchus contortus; & Decreasing parasitaemia & $74.07 \%$ & [24] \\
\hline & Babesia microti against $P$. cynomolgi & & & \\
\hline \multirow{10}{*}{$\begin{array}{l}\text { Between Parasites } \\
\text { and bacteria }\end{array}$} & Enterobacter amnigenus against $P$. vivax ${ }^{c}$ & Inhibiting transmission & $94.37 \%$ & [25] \\
\hline & Enterobacter cloacae against $P$. vivax ${ }^{c}$; & Inhibiting transmission & $83.10 \%$ & [25] \\
\hline & Serratia marcescens against $P$. vivax ${ }_{i} ;$ & Inhibiting transmission & $98.59 \%$ & [25] \\
\hline & Ewingella americana against $P$. falciparum ${ }^{c}$ & Inhibiting transmission & $38.00 \%$ & {$[26]$} \\
\hline & Salmonella typhimurium against Schistosoma; & $\begin{array}{l}\text { Decreasing parasitaemia } \\
\text { and attenuating symptoms }\end{array}$ & $56.82 \%$ & [27] \\
\hline & Giardia muris against Citrobacter rodentium; & Inhibiting propagation & $42.86 \%$ & [28] \\
\hline & Wolbachia (wMelPop strain) against $P$. falciparum ${ }^{c}$ & Decreasing parasitaemia & $46.78 \%$ & {$[29]$} \\
\hline & Wolbachia (wMelPop strain) against $P$. berghei ${ }^{c}$ & Decreasing parasitaemia & $33.00 \%$ & [11] \\
\hline & Wolbachia (WMelPo strain) against P. gallinaceum ${ }^{c}$ & Inhibiting propagation & $79.50 \%$ & [30] \\
\hline & & & $80.00 \%$ & \\
\hline \multirow{3}{*}{$\begin{array}{l}\text { Between Parasites } \\
\text { and Viruses }\end{array}$} & Plasmodium against chikungunya virus; & Attenuating symptoms & $39.50 \%$ & {$[31,32]$} \\
\hline & Heligmosomoides polygyrus against respiratory syncytial virus; & Attenuating symptoms & $42.06 \%$ & {$[33]$} \\
\hline & Echinoparyphium against ranavirus & Decreasing viral loads & $23.50 \%$ & [34] \\
\hline
\end{tabular}

${ }^{a}$ Contradictory results

${ }^{\mathrm{b}}$ Antagonism between different strains of $P$. berghei

'Laboratory conditions

${ }^{\mathrm{d} C o n d i t i o n s}$ in human population

against infection" phenomena could possibly contribute to the development of a novel prevention strategy for infectious diseases.

\section{Parasites against parasites}

Nematodes are widely distributed parasites that adapt successfully to nearly every ecosystem, and they are able to suppress the invasion, development and reproduction of certain parasites from other species. An animal study demonstrated decreased Plasmodium berghei parasitaemia in adult mice coinfected with Trichinella spiralis. This enhanced resistance to malaria was partially attributed to the nematode-induced activation of the mononuclear phagocyte system and a decrease in reticulocyte levels [15]. Additional evidence provided by another retrospective study indicated that malaria patients previously infected with helminths, such as Ascaris lumbricoides, T. trichiura, hookworm and Strongyloides stercoralis, were less likely to have mature schizonts in their peripheral blood or tissues or to suffer from renal failure, jaundice and severe cerebral malaria than those without protective helminth infections $[16,17,35]$. Moreover, two randomized trials identified a negative interaction between $A$. lumbricoides and Plasmodium falciparum [36, 37], while another trial in Nigeria showed a contrasting result [38]. Similar protection also occurred between filarial parasites and malarial parasites. To see this, consider an example involving BALB/c mice coinfected with Litomosoides sigmodontis and P. berghei. Mice did not develop blood-stage malaria [22], but their condition deteriorated unless filarial infection achieved patency [39]. Another example is the significant reduction in the intensity of malarial attack caused by the co- 
occurrence of Brugia malayi and B. pahangi infection [40]. Despite the protection of filarial parasites against Plasmodium, synergism was observed between filarial and other protozoa (Wuchereria bancrofti and malarial parasites, B. malayi and Leishmania donovani) [41, 42]. In addition, other studies confirmed that nematodes blocked the transmission of some trematodes by regulating the population of fresh water snails and intermediate hosts. For instance, after being transferred into snails, Heterorhabditis baujardi induced parasitic castration and consequently contributed to an average death rate of $67 \%$ in Lymnaea columella, the intermediate host of Fasciola hepatica and Fasciola gigantica. Therefore, $H$. baujardi was expected to become a feasible alternative for the prevention of fasciolosis [12]. Similarly, parasitic castration also occurred in Biomphalaria glabrata experimentally infected with Angiostrongylus cantonensis [13]. Although nematodes had the potential to suppress other parasitic infections, the interaction between coinfecting nematodes was intriguing and required further study. Pre-existing Trichostrongylus colubriformis negatively affected the survival of Haemonchus contortus, but contradictory results occurred with different infection orders [23]. The primary pathogen infecting the host was likely a determining factor in the relationship between Tetracapsuloides bryosalmonae and Myxobolus cerebralis [43], but obvious antagonism was found between another two protozoa, Babesia microti and Plasmodium cynomolgi [24]. Interestingly, other studies indicated a positive association between concurrent protozoa in exacerbating clinical manifestation $[44,45]$ or increasing susceptibility of the hosts to the other protozoan, implying that in-depth studies are needed to clarify interactions between coinfecting protozoa [46]. In human populations, some studies have been conducted to generate an in-depth understanding of the interaction between concomitant infections with malaria parasites and Schistosoma, but these studies showed contradictory results. Some studies suggested a protective role for helminths [18-21, 47-50]; for example, one crosssectional study demonstrated lower $P$. falciparum parasite densities in children with mild S. haematobium infection than those in the control group [18, 19], and a further study suggested an age-dependent manner of the protective effect [47]. Animal experiments also reported similar results where parasite growth and gametocyte infectivity of Plasmodium yoelii or Plasmodium knowlesi were both inhibited by coinfection with Schistosoma mansoni $[20,21]$. However, other studies claimed that concurrent exposure to $P$. falciparum and $S$. mansoni might be synergistically associated in elevating parasitaemia, [51-53] exacerbating clinical manifestations [54-56] and increasing infection risks [57-60].

Surprisingly, an antagonistic interaction was also identified between diverse strains of malaria parasites. The total parasite densities in mice infected with virulent strains of $P$. berghei were reduced if mildly infective strains were inoculated into the hosts in advance [61].

\section{Parasites against viruses}

Parasites were also found to reduce the severity of viral infections. Giardia lamblia might modulate the pathogenicity of rotaviruses if concurrent infections occur [62]. Compared with rotavirus monoinfections, G. lambliarotavirus coinfections triggered diarrhoeal episodes of reduced severity [62]. A similar protective effect was discovered between Plasmodium and chikungunya virus (CHIKV) [31, 32]. Moreover, in animal experiments, nematodes such as T. spiralis and Nematospiroides dubius showed the ability to alleviate pathological changes caused by influenza A, while T. spiralis could suppress inflammatory infiltration in the lungs and downregulate cytokine production in the bronchoalveolar lavage fluid, and $N$. dubius was demonstrated to reduce viral titres in the lungs $[35,63]$. Similar protection was discovered in Heligmosomoides polygyrus-infected mice, which demonstrated significantly attenuated pulmonary diseases after respiratory syncytial virus (RSV) [33]. In addition to protozoa and nematodes, trematodes (Echinoparyphium) were also negatively associated with viruses (ranavirus) by decreasing virus loads [34]. Conversely, other helminths were synergistically connected with some viruses by accelerating disease progression (lymphocytic choriomeningitis virus and Leishmania guyanensis, Toscana virus and L. guyanensis, Friend virus and L. sigmodontis, hepatitis $C$ virus and S. mansoni) [64-66] or in increasing virus loads [67]. However, the relationship between human immunodeficiency virus (HIV) and parasites remains unclear. Some studies demonstrated that viral replication was diminished under Trypanosoma cruzi infection, while more evidence suggested that coinfection with parasites promotes viral replication [68], cell-to-cell transmission of virus [69] and exacerbation of clinical manifestations [70, 71].

\section{Parasites against bacteria}

Malaria is a mosquito-borne infectious disease that has captured the attention of international health organizations. According to the World Malaria Report, in 2017, 216 million cases were reported in 91 countries, and the global death toll reached 445000 . The elimination of malaria has been urgently advocated by the WHO [2]. The key to eliminating malaria is blocking the transmission of malaria parasites via the malarial vector, namely, the Anopheles mosquito. The life cycle of malaria parasites might be inhibited by many bacteria, for example, enterobacteria. When Enterobacter amnigenus, Enterobacter cloacae and Serratia marcescens were transferred along with $P$. vivax-infected blood into Anopheles albimanus, the infection rate of the coinfected mosquitoes 
decreased significantly compared with that of the control group; notably, the mean oocyst level in mosquitoes coinfected with $E$. cloacae was 2.5 times lower than that of the control group [25]. Moreover, coinfection with $S$. marcescens resulted in a decrease in the mosquito mortality rate [25]. By the same token, the formation of $P$. falciparum oocysts in A. stephensi was reported to be hindered by coinfection with gram-negative bacteria, such as Escherichia coli, Pseudomonas aeruginosa and Ewingella americana [26]. Another study showed a reduction in the number of adult schistosomal worms and eggs and a relief of schistosomiasis symptoms during coinfection with Salmonella typhimurium [27]. In regard to coinfection with $P$. yoelii, the susceptibility of hosts to S. typhimurium increased [72]. In contrast, the growth of enterobacteria (Citrobacter rodentium) was suppressed by coinfection with Giardia muris or Giardia duodenalis [28].

In addition to enterobacteria, Wolbachia pipientis, bacteria mainly infecting arthropods and nematodes, performed an essential function in malaria control. Somatic infections of Anopheles gambiae with the wMelPop strain of Wolbachia led to decreased oocyst levels and parasitic densities of P. falciparum [29] and P. berghei [11]. Furthermore, the wAlbB strain of Wolbachia induced P. falciparum resistance in Anopheles stephensi, a major vector of malaria parasites in South Asia and the Middle East [10]. Similar interactions were observed between Wolbachia (wMelPop strain)-infected Aedes aegypti and P. gallinaceum [8]. Interestingly, the blocking of malaria parasites by Wolbachia was modulated by temperature [30]. A model system of P. yoelii and A. stephensi revealed a reduction in both parasitic prevalence and oocyst intensity at $28{ }^{\circ} \mathrm{C}$, unchanged parasitic prevalence and increased oocyst intensity at $24{ }^{\circ} \mathrm{C}$, and unchanged parasitic prevalence and unchanged oocyst intensity at $20{ }^{\circ} \mathrm{C}$ [30].

Wolbachia played vital roles in vector modulation for the control of filariasis in tropical and subtropical areas. The infection of $A$. aegypti with Wolbachia prior to infection with $B$. pahangi microfilariae induced striking reductions in both the prevalence of infected mosquitoes and the mean number of infective parasites [9]. Nevertheless, additional evidence is needed to demonstrate the potential ability of Wolbachia to treat filariasis in humans in consideration of $B$. pahangi's role as a filarial nematode in rodents. In general, studies on Wolbachia have provided exciting prospects for the elimination of mosquito-borne diseases, such as malaria and dengue.

Interactions between Mycobacterium tuberculosis and other concurrent pathogens were also highly studied. With current studies, we can temporarily conclude a synergistic relationship between $M$. tuberculosis and helminths in disease progression [73-75].
In conclusion, the antagonistic association between parasites and other concurrent pathogens was manifested as reduced susceptibility of hosts, growth suppression, and decreased transmission or attenuated clinical manifestations. The intriguing findings of antagonism between helminths and other various pathogens increase the comprehensive understanding of the mutualistic relationships between different pathogens. Elucidation of the mechanisms behind these antagonistic interactions is necessary; this knowledge will be conducive to controlling infectious diseases with a higher efficiency.

\section{Mechanism of parasite antagonism against parasites, viruses and bacteria \\ Apparent competition: Immunomodulation}

Coinfection is a common occurrence that predominantly alters disease progression. Due to the shared endemicity of pathogens in vast areas, coinfection between pathogens, helminths in particular, has been expected and feared [4, $76,77]$. Despite the limited number of available studies, the preliminary conclusion that interactions between helminths and other pathogens seem to be complex and bidirectional can be safely drawn [78-80]. Currently, studies suggest that helminth infection could lead to robust cytokine production that could promote the occurrence of malaria $[19,47,48,78,79,81]$. Nevertheless, there is clinical and epidemiologic evidence to support the notion that helminth infection favours protection by reducing the density of pathogens and mitigating immunopathological injury [81]. Studies have been conducted in models of experimental cerebral malaria (ECM) to explore the underlying immunological mechanisms [14, 79]. The outcomes not only rested on the different genetic backgrounds of the hosts but also depended on the species and densities of the pathogens [82].

A bimodal immune response is the distinctive characteristics of malaria containing a Th1-type response for control of the initial parasitaemia and further Th2-mediated cytokine production for parasite clearance $[4,83]$. Helminth infections, known as potent inducers of the Th2-type response, can downregulate the effects of a secondary Th1-dependent parasitic challenge [84-87].In the absence of the initial inflammatory stage, $\mathrm{T}$ cellmediated antigen clearance would be blocked, and the rapid proliferation of pathogens would ensue. Additionally, uncontrollable inflammatory reactions could induce serious immunopathological lesions. Supplementary studies have confirmed that the robust Th2-type response induced by chronic helminth infection could suppress the proinflammatory Th1-type response [88-90]. The emphasis of discussion in this section revolves mainly around Schistosoma, such as $S$. mansoni [91-93], S. japonicum [94, 95] and S. haematobium $[18,96,97]$. In the context of a low burden of malaria 
parasites along with an increase in Schistosome cercariae, the protective antimalarial immune response would be enhanced [84]. Several potential mechanisms might induce the negative interaction between these players $[98,99]$. However, no significant interaction between malaria parasites and Schistosome was observed as a result of challenge with a heavy malaria parasite burden during coinfection, and its causes remain unclear [84].

\section{Suppression of dendritic cell $(D C)$ responses to competitors}

DC subgroups in the spleen mainly include plasmacytoid DCs (pDCs) and myeloid DCs (mDCs). Numerous studies have confirmed that DCs are able to induce the adaptive immune response in the initial phase of malaria parasite infection, during which the predominant Th1 immune response induces the development of cerebral malaria. Mature DCs are critical antigen-presenting cells (APCs) that activate the Th1-type response and cytotoxic $\mathrm{T}$ cell response. In the early stage of malaria, phagocytosis by DCs is enhanced to selectively identify and remove pRBCs. The activation of naive T cells and the polarization switch from Th0 to Th1 are closely related to the mature DC phenotype, which is marked by the upregulation of major histocompatibility complex (MHC) class II molecules and the CD80/CD86 costimulatory molecules. The high-level expression of MHC class II molecules expedites the processing and presentation of malaria parasite antigens by DCs to activate CD4+ T cells and hence trigger the Th1 and cytotoxic T lymphocyte responses. The activation of $\mathrm{CD} 8+\mathrm{T}$ cells by malaria parasite infection may further jeopardize the microvascular endothelial tissue and blood-brain barrier (BBB) through the activity of the perforin/granzyme pathway [100] and the LT- $\alpha$ pathway [101], which in turn allows malaria parasite components and other potential damage factors to enter the brain parenchyma. Consequently, the overactivation of glial cells is induced, and the apoptosis of astrocytes becomes uncontrolled. During monoinfection of malaria pathogens, a striking increase in the vascular permeability of the brain tissue was observed [94]. However, during challenges by relatively low densities of malaria parasites in the coinfection, increased helminth loads contributed to an apparent decrease in the counts of mature DCs along with a further decrease in functional DC responses to induce a Th1 predisposition, thereby providing crucial protection against ECM pathogenesis [19, 37, 47, 94, 102].

Exposure to helminths not only dampens the maturation of DCs but also promotes the population expansion of regulatory $\mathrm{T}$ (Treg) cells. Immature DCs can produce IL-10 and/or TGF- $\beta$ in response to exposure to helminth excretory-secretory products. In vitro experiments demonstrated that IL-10 and TGF- $\beta$ were essential cytokines that induced the differentiation and amplification of Treg cells from naive $\mathrm{T}$ cell precursors and promoted the further expansion of pre-existing Treg cell subpopulations [61]. The dynamic balance between proinflammatory and anti-inflammatory responses is indispensable for maintaining homeostasis. After the proinflammatory Th1-type response is established in the early stage of parasitic infection, specific antibodies produced by plasma cells with the help of Th2 cells could efficaciously remove worms and prevent disease recurrence [94]. Treg cells, such as CD4+CD25+Foxp3+, are capable of maintaining proper immune homeostasis by regulating the production of proinflammatory and antiinflammatory cytokines and by mediating the differentiation of naive $\mathrm{T}$ cells into diverse subgroups, such as Th1, Th2 and Treg cells [47, 88].

\section{Activation of basophils and mononuclear macrophages against competitors}

During the occurrence and progression of cerebral malaria, the deposition of pRBCs in cerebral capillaries causes the adhesion of immune cells and platelets to vascular endothelial cells, thus resulting in encephalorrhagia, hydrocephalus and $\mathrm{BBB}$ permeability changes. Among them, the infiltration of $\mathrm{T}$ cells (especially $\mathrm{CD} 8+\mathrm{T}$ cells) is critical for the increase in the permeability of the $\mathrm{BBB}$ and brain tissue injury. The excessive release of proinflammatory cytokines, chemokines and adhesion molecules is closely related to the above process, especially IFN- $\gamma$.

With the demonstration of a positive correlation between the burden of schistosomal infection and Th2 cytokine synthesis, helminth-induced activation of basophils and mononuclear macrophages may be the critical mechanism underlying the Th2-type response bias and alleviation of BBB impairment [103].

In response to schistosomal antigens, such as IPSE $/ \alpha-$ 1 , a major secretory glycoprotein antigen from eggs of Schistosome, the notable upregulation of Th2 cytokines was detected, IL-4 and IL-10 [104] in particular, suggesting that in the Treg-mediated anti-inflammatory response, IL-4 and IL-10 might predominate in inhibiting the Th1-type response in malaria [94, 105], and IL-4 could suppress the IFN- $\gamma$-secreting Treg cells. The Th1biased differentiation of $\mathrm{T}$ cells into IFN- $\gamma$-producing effectors was markedly down-modulated in response to IL-4 treatment [103]. The reduction in IFN- $\gamma$ caused the production of chemokines (such as CXCL9 and CXCL10) and adhesion molecules (such as ICAM-1), the sequestration and deposition of parasite-infected red blood cells (pRBCs), leukocytes and platelets, and the migration of $\mathrm{CD} 8+\mathrm{T}$ cells into the brain to be restrained [105]. Furthermore, IL-4 could activate GATA3, the specific transcription factors of Th2 cells, by signalling pathways (e.g., STAT6 pathway, transcription factors, even self-regulation). Conversely, GATA3 promoted the 
positive regulation of IL-4 production in Th2 cells and led to a positive-feedback loop. In accordance with the previous situation, significant changes were not observed in severe malarial infections [91]. With the exception of basophils and monocytes, antigens alternatively activated M2 macrophages, which then secreted IL-10 and other anti-inflammatory cytokines. IL-10, in contrast to IL-4, might target Th1 effector functions by preventing the production of IFN- $\gamma[106]$. Likewise, Th2 cells release the relevant cytokines to drive M2 polarization of macrophages to form the positive-feedback effect [107]. Regarding Schistosoma infection, the bulk of the reported data from ECM models indicated a decrease in the expression level of mRNAs to be translated into proinflammatory factors associated with brain histopathological characteristics. Furthermore, alterations in the parasite density could result in corresponding alterations in the above parameters [91]. Alleviation of microvascular lesion formation and BBB permeability provided noteworthy improvements in symptom severity by reducing the activation of CD8+ T cells in the brain and alleviating neurotoxicity [95].

Consistent with the results of a series of previous studies, the immune environment of chronic helminth infection appears to favour the induction of counter-regulatory responses suppressing infection by other pathogens [83]. Several reports have revealed that helminth infection is closely involved in the decrease in viral burden in peripheral and placental blood [108, 109].

\section{Adjuvant effect of complement system}

It has been previously confirmed that the C3d component of the complement system acts as a bridge between innate and acquired immunity. The adjuvant effect of C3d has been exemplified in S. mansoni models [96]. It was found that C3d, a breakdown product of complement C3, was present in high amounts. C3d, containing the ligand for CD21, a B cell co-receptor, would be expected to bind to CR2 (CD21) to promote the development and maintenance of memory $B$ lymphocytes. Different hypotheses have been developed to explain this function, including the tyrosine-phosphorylated association of CD21 with CD19 and the CD21 expression of follicular dendritic cells $[96,110,111]$.

Under the influence of C3d, the complement system could select antigens for recognition by the acquired immune system and be complementary to some other components of innate immunity to notably raise the capability of anti-infection in hosts.

The above contents have been thoroughly studied at present. In addition, Toll-like receptors (TLRs) have been clarified to participate in antimalarial defence. While TLR9 responds to malaria pigments, TLR4 responds to glycosylphosphatidylinositol (GPI) produced by $P$. falciparum. However, the idiographic mechanism of action underlying these responses has not been elucidated. TLR-mediated immunopathological damage of brain tissue is linked to the aggregation of CD8+ T cells, DCs, and natural killer (NK) cells. Experimental research has already disclosed the downregulated expression of TLRs in the presence of Schistosoma cercariae [96]. The specific mechanism still needs further exploration.

Coincidentally, antibody-mediated immune modulation also occurred in the interactions between some pathogens. Higher levels of Clonorchis sinensis-specific IgG and IgA elicited upon $T$. spiralis infection have been the subject of several reports, indicating that enhanced C. sinensis clearance induced by coinfection was associated with systemic and mucosal IgG and IgA responses [112, 113].

\section{Exploitation competition: Resource limitation}

If two or more organisms have similar needs for limited resources and space, fierce competition for survival opportunities and reproductive capacity enhancements will inevitably ensue $[4,114,115]$. Competition occurs at times in a vast geographic distribution, and the environment of coinfection is no exception.

If the struggle for existence among these organisms persists long enough, adaptive evolution might occur in order for each organism to occupy a favourable position. This process, termed exploitation competition, is divided into two strategies: divergent resource use between the organisms when suitable alternative resources are available and adaptations that improve the ability to acquire those shared resources when there are no alternatives [116, 117]. Taking advantage of exploitation competition is devastating to competitors. Tissue tropism is believed to explain the phenomenon that some species of trematodes prefer to infect a particular host tissue. Despite evidence indicating the tropism of Diplostomum hupehensis to the intestine of the avian host and to different eye tissues of the fish host [116], the direct demonstration of the underlying mechanism remains unknown thus far. However, the latter exploitation competition strategy is more applicable, especially in regions of high malaria endemicity [114]. The mutation of enzymes could drive the conversion of Plasmodium stages to favour the asexual stages over the single sexually differentiated stages, thus simultaneously supporting both the reduced transmission efficiency and the increased infection efficiency. A rosetting phenotype in which uninfected cells adhere to a central infected cell has been observed. Pathogens might be expected to burst from the central infected cell and specifically target peripheral cells in rosettes, but this reasonable hypothesis has not yet been tested [118].

\section{Interference competition: Direct attack or exclusion}

In addition to the abovementioned mechanisms, coinfection might enter into interference competition by 
producing toxic compounds that directly eliminate or hinder the development of pathogenic competitors [61, $118,119]$.

Epidemiological and experimental studies have revealed that intestinal symbiotic bacteria could stimulate the innate immune system of arthropods and upregulate the expression of multiple genes inhibiting other infections, such as cecropins, defensins and gambicin $[28,61$, $118,120]$. Interference competition also occurred in certain cestodes, which could release substances termed "crowding factors" [116]. Crowding factors are arguably the least ambiguous interference strategy: they seem to be explained as a passive accumulation of end metabolites serving as a signal for worms to slow their growth; however, crowding factors have adaptive value in moderating the proliferation of competitors when resources are limited. Regretfully, research on crowding factors was tortuous, progressed slowly and has gradually been ignored in recent years $[21,121]$. Hence, clarification of this uncertainty remains elusive.

References to biological control of disease transmission in intermediate hosts or vectors Abundant epidemiological investigations and experimental studies suggested that several nematode species could interrupt the expansion of infectious diseases by reducing or even obliterating the reproductive capacity of intermediate hosts or vectors. This suggestion appeared to be borne out by recent research on entomopathogenic nematodes (EPNs) and symbiotic bacteria. EPNs carry symbiotic bacteria, which, after the EPNs enter the host, are released in the intestinal cavity [122] and produce a fatal neurotoxic metabolite. The nematodes then reproduce in the dead host, and the new generation of offspring invades the next host [123]. The role of EPNs in snails needs further exploration. The biological control of intermediate hosts or vectors is likely to confer considerable prophylactic and therapeutic benefits [124].

\section{Proteolysis and inversion in the excretion pattern}

Numerous experimental results noted a significant decrease in the concentration of total protein in infected snails, which occurred gradually as the infection advanced. A case in point is the infection of B. glabrata by A. cantonensis. The microbiological interaction involved was accompanied by a substantial increase in the level of urea and a significant reduction in the concentration of uric acid in the haemolymph derived from infected specimens [125], suggesting that nematode infections induce proteolysis and an inversion in the excretion pattern of infected snails. According to the related enzymatic investigation, the activity levels of alanine aminotransferase (ALT) and aspartate aminotransferase (AST) were markedly higher in the infected groups than in the control group. These changes produced an increasing rate of amino acid deamination, thus supplying alternative substrates for gluconeogenesis. Moreover, the accumulation of urea accelerated the urea cycle; thus, more arginine was available for parasite development [121]. Interestingly, the nitrogen degradation products could produce noxious effects on the host neuroendocrine system and even kill the infected snails, thus creating a comfortable environment for nematodes to accomplish larval-stage development. The reduction in the population of intermediate hosts carrying pathogens has a positive impact on inhibiting the transmission of infectious diseases, especially in natural epidemic foci (e.g., the transmission of angiostrongyliasis in Guangdong, China) $[122,126]$.

\section{Degenerative capacity}

Current research has demonstrated that nematodes weaken the reproductive capacity of snails mainly by direct impairment of the reproductive tissues and organs or by indirect harm through the withdrawal of nutrients $[12,13]$. The former mechanism suggested that nematodes induced egg granuloma formation and fibrosis, rendering the snail reproductive organs vulnerable. Over time, the inflammatory infiltration and fibrosis within cephalopods hindered motor function, thus affecting the feeding and fertilization abilities of snails. The latter mechanism speculated that nematodes competed with the host to obtain essential nutrients for proliferation, the outcome of which was the suppression of the reproductive capacity of the snails. The galactogen synthesized by the albumen gland, for instance, served as the primary energy source for snail embryos and newly hatched offspring. A galactogen deficiency in the albumen gland would decelerate the hatching rate of infected snails, thus characterizing parasitic castration as a nutritional process [12].

\section{Suppressive effect on transmission}

Wolbachia is a renowned intracellular endosymbiont of invertebrates that is capable of protecting insects from pathogens and limiting their ability to transmit mosquitoborne pathogens by the reproductive manipulation of their hosts by means such as sperm-egg cytoplasmic incompatibility $(\mathrm{CI})$, which results in the production of unviable progeny when a male mosquito carrying Wolbachia mates with a wild female mosquito [127, 128]. The above interaction was reported to occur during the successful introduction of Wolbachia into the mosquito species (e.g., Aedes, Anopheles and Culex), which are the major vectors of human pathogens, including protozoa (Plasmodium sp.), filariae and a variety of viruses (causing dengue, yellow fever, and West Nile) [129]. Specialists were inspired to apply this mechanism of antagonism in the venues for malaria control. 
The development of various pathogens in mosquitoes was confirmed to be inhibited by immune preactivation, thus suggesting that constitutive immunoregulation could influence the transmission of infectious pathogens to humans [124]. A more in-depth study conducted recently showed the capability of Wolbachia to induce immune system upregulation. Wolbachia stimulated mosquitoes to produce reactive oxygen species (ROS) in testes and ovaries; this ROS production later activated the Toll pathway and resulted in the synthesis of a variety of antibacterial peptides, such as defensins and cecropins, thus suppressing other coinfected pathogens $[9,130]$.

Another striking example of this surprising ability of Wolbachia is that the over-replicating wMelPop strain of Wolbachia could induce the constitutive expression of innate immune genes in mosquitoes [9], thus implying a potential explanation for the interspecific competitive relationships between different parasites as a contributory factor to the life-shortening parasitic phenotypes [106, 131].

\section{Conclusions}

Previous studies have illuminated the antagonism between parasites and parasites, parasites and viruses, and parasites and bacteria (Table 1). Further research has described four possible mechanisms underlying this phenomenon. In particular, the immunomodulation theory, which indicates that coinfection was able to reduce the immunopathological alterations caused by the Th1type immune response, has been widely accepted $[4,91]$. A low intensity of Schistosoma infection could improve the protective antimalarial immune response associated with the expression of related cytokines [18, 91, 94, 132], the permeability of the BBB [94], the DC response [94], and the differentiation and activation of Tregs [94], thereby potentially being protective against cerebral malaria. Vector modulation is another universally acknowledged hypothesis to explain antagonistic coinfection; quintessential examples of vector modulation include the effects of EPNs and Wolbachia [12, 13, 106, 123, $125,127,128,133]$. Other mechanisms underlying antagonism include interference competition and exploitation competition $[61,116,134]$.

In summary, antagonistic phenomena between coinfecting pathogens are common, but persistent efforts are required to elucidate the underlying mechanisms and identify more effective strategies for combating pathogenic infection $[77,135]$. Though antagonism involving parasites has been highlighted in this review, the unexpected "infection against infection" relationship has also been observed in the cotransmission of bacteria and viruses. One of the most promising examples is that the Wolbachia strains that had been successfully established in wild Ae. Aegypti to control dengue virus are now undergoing field trials in dengue endemic areas of Australia, Brazil, Indonesia and Vietnam [136]. The "infection against infection" relationship will offer novel control strategies for infectious diseases, such as biological control of parasitic vectors and potent vaccine development.

\section{Additional file}

Additional file 1: Multilingual abstracts in the five official working languages of the United Nations. (PDF $345 \mathrm{~kb}$ )

\section{Abbreviations}

ALTA: lanine aminotransferase; AMR: Antimicrobial resistance; APC: Antigenpresenting cell; AST: Aspartate aminotransferase; BBB: Blood-brain barrier; Cl: Cytoplasmic incompatibility; DC: Dendritic cell; ECM: Experimental cerebral malaria; EPN: Entomopathogenic nematode; GPI: Glycosylphosphatidylinositol; mDC: Myeloid dendritic cell; MHC: Major histocompatibility complex; NK: Natural killer cell; pDC: Plasmacytoid dendritic cell; pRBC: Parasite-infected red blood cell; ROS: reactive oxygen species; TLR: Toll-like receptor;

Treg: Regulatory T cell; WHO: World Health Organization

Acknowledgements

We sincerely appreciate Prof. Mingchiu Fung for his reviewing the manuscript

\section{Authors' contributions}

SSS, XYQ and WZZ carried out the literature search and drafted the first version of the manuscript. $J L$ and $Z Y L$ contributed to conceive the review concept and revised the manuscript. All authors have read and approved the final manuscript.

\section{Funding}

This work was supported by the Project of Basic Platform of National Science and Technology Resources of the Ministry of Sciences and Technology of China (No. TDRC-2017-22), the National Key R\&D Program of China (No. 2016YFC1202003, 2016YFC1202005 and 2016YFC1200500), the National Natural Science Foundation of China (No. 81371836, 81572023 and 81271855), and the 111 Project (No. B12003).

Availability of data and materials

The datasets used and/or analyzed during the current study are available from the corresponding author upon reasonable request.

Ethics approval and consent to participate

Not applicable.

Consent for publication

Not applicable.

Competing interests

The authors declare that they have no competing interests.

Author details

'Zhongshan School of Medicine, Sun Yat-sen University, Guangzhou 510080, China. ${ }^{2}$ Fifth Affiliated Hospital, Zhongshan School of Medicine, Sun Yat-sen University, ZhuHai, Guangdong, China. ${ }^{3}$ Key Laboratory of Tropical Disease Control, Ministry of Education, Sun Yat-sen University, Guangzhou, China. ${ }^{4}$ Provincial Engineering Technology Research Center for Biological Vector Control, Guangzhou, China.

Received: 19 November 2018 Accepted: 28 May 2019 Published online: 15 June 2019

\section{References}

1. The World Health Organization. http://www.who.int/en/news-room/factsheets/detail/the-top-10-causes-of-death. Accessed 24 May 2018.

2. The World Health Organization. http://www.who.int/en/news-room/factsheets/detail/antimicrobial-resistance. Accessed 15 Feb 2018. 
3. Bustinduy AL, Sutherland LJ, Chang-Cojulun A, Malhotra I, DuVall AS, Fairley JK, et al. Age-stratified profiles of serum IL-6, IL-10, and TNF-alpha cytokines among Kenyan children with Schistosoma haematobium, Plasmodium falciparum, and other chronic parasitic co-infections. Am J Trop Med Hyg. 2015;92:945-51.

4. Griffiths EC, Pedersen AB, Fenton A, Petchey OL. Analysis of a summary network of co-infection in humans reveals that parasites interact most via shared resources. Proc Biol Sci. 2014;281:20132286.

5. Bourtzis K, Dobson SL, Xi Z, Rasgon JL, Calvitti M, Moreira LA, et al. Harnessing mosquito-Wolbachia symbiosis for vector and disease control. Acta Trop. 2014;132:S150-63.

6. Jeffries $\mathrm{CL}$, Walker T. The potential use of Wolbachia-based mosquito biocontrol strategies for Japanese encephalitis. Plos Negl Trop Dis. 2015;9:e0003576.

7. LePage D, Bordenstein SR. Wolbachia: can we save lives with a great pandemic? Trends Parasitol. 2013;29:385-93.

8. Walker T, Moreira LA. Can Wolbachia be used to control malaria? Mem Inst Oswaldo Cruz. 2011;106(Suppl 1):212-7.

9. Kambris Z, Cook PE, Phuc HK, Sinkins SP. Immune activation by lifeshortening Wolbachia and reduced filarial competence in mosquitoes. Science. 2009;326:134-6.

10. Bian G, Joshi D, Dong Y, Lu P, Zhou G, Pan X, et al. Wolbachia invades Anopheles stephensi populations and induces refractoriness to Plasmodium infection. Science. 2013;340:748-51.

11. Kambris Z, Blagborough AM, Pinto SB, Blagrove MSC, Godfray HCJ, Sinden RE, et al. Wolbachia stimulates immune gene expression and inhibits Plasmodium development in Anopheles gambiae. Plos Pathog. 2010;6:e1001143.

12. Tunholi VM, Lorenzoni PO, da Silva YH, Tunholi-Alves VM, Boeloni JN, da Silva MA, et al. Molluscicidal potential of Heterorhabditis baujardi (Rhabditida: Heterorhabditidae), strain LPP7, on Lymnaea columella (Gastropoda: Pulmonata): An alternative for biological control of fasciolosis. Acta Trop. 2017;173:23-9.

13. Tunholi-Alves VM, Tunholi VM, Lustrino D, Amaral LS, Thiengo SC, Pinheiro J. Changes in the reproductive biology of Biomphalaria glabrata experimentally infected with the nematode Angiostrongylus cantonensis. J Invertebr Pathol. 2011:108:220-3.

14. Nacher $M$, Singhasivanon $P$, Silachamroon $U$, Treeprasertsuk $S$, Vannaphan $S$, Traore $B$, et al. Helminth infections are associated with protection from malaria-related acute renal failure and jaundice in Thailand. Am J Trop Med Hyg. 2001;65:834-6.

15. Ngwenya BZ. Enhanced resistance to Plasmodium berghei in mice previously infected with Trichinella spiralis. Parasite Immunol. 1982:4:197-207.

16. Nacher $M$, Singhasivanon $P$, Treeprasertsuk S, Vannaphan S, Traore B, Looareesuwan S, Gay F. Intestinal helminths and malnutrition are independently associated with protection from cerebral malaria in Thailand. Ann Trop Med Parasitol. 2002;96:5-13.

17. Abbate JL, Ezenwa VO, Guegan JF, Choisy M, Nacher M, Roche B. Disentangling complex parasite interactions: protection against cerebral malaria by one helminth species is jeopardized by co-infection with another. Plos Negl Trop Dis. 2018;12:e0006483.

18. Lemaitre M, Watier L, Briand V, Garcia A, Le Hesran JY, Cot M. Coinfection with Plasmodium falciparum and Schistosoma haematobium: additional evidence of the protective effect of Schistosomiasis on malaria in Senegalese children. Am J Trop Med Hyg. 2014;90:329-34.

19. Briand V, Watier L, JY LEH, Garcia A, Cot M. Coinfection with Plasmodium falciparum and Schistosoma haematobium: protective effect of schistosomiasis on malaria in Senegalese children? Am J Trop Med Hyg. 2005;72:702-7

20. Nyakundi RK, Nyamongo O, Maamun J, Akinyi M, Mulei I, Farah IO, et al. Protective effect of chronic schistosomiasis in baboons coinfected with Schistosoma mansoni and Plasmodium knowlesi. Infect Immun. 2016;84: 1320-30.

21. Moriyasu T, Nakamura R, Deloer S, Senba M, Kubo M, Inoue M, et al. Schistosoma mansoni infection suppresses the growth of Plasmodium yoelii parasites in the liver and reduces gametocyte infectivity to mosquitoes. Plos Negl Trop Dis. 2018;12:e0006197.

22. Fernandez Ruiz D, Dubben B, Saeftel M, Endl E, Deininger S, Hoerauf A, et al. Filarial infection induces protection against $P$. berghei liver stages in mice. Microbes Infect. 2009;11:172-80.

23. Lello J, McClure SJ, Tyrrell K, Viney ME. Predicting the effects of parasite coinfection across species boundaries. Proc Biol Sci. 2018;285:20172610.

24. van Duivenvoorde LM, Voorberg-van der Wel A, van der Werff NM, Braskamp G, Remarque EJ, Kondova I, et al. Suppression of Plasmodium cynomolgi in rhesus macaques by coinfection with Babesia microti. Infect Immun. 2010:78:1032-9.

25. Gonzalez-Ceron L, Santillan F, Rodriguez MH, Mendez D, Hernandez-Avila JE. Bacteria in midguts of field-collected Anopheles albimanus block Plasmodium vivax sporogonic development. J Med Entomol. 2003;40:371-4.

26. Pumpuni CB, Beier MS, Nataro JP, Guers LD, Davis JR. Plasmodium falciparum: inhibition of sporogonic development in Anopheles stephensi by gram-negative bacteria. Exp Parasitol. 1993;77:195-9.

27. Zhu X, Chen L, Wu J, Tang H, Wang Y. Salmonella typhimurium infection reduces Schistosoma japonicum worm burden in mice. Sci Rep. 2017;7:1349.

28. Manko A, Motta JP, Cotton JA, Feener T, Oyeyemi A, Vallance BA, et al. Giardia co-infection promotes the secretion of antimicrobial peptides betadefensin 2 and trefoil factor 3 and attenuates attaching and effacing bacteria-induced intestinal disease. Plos One. 2017;12:e0178647.

29. Hughes GL, Koga R, Xue P, Fukatsu T, Rasgon JL. Wolbachia infections are virulent and inhibit the human malaria parasite Plasmodium falciparum in Anopheles Gambiae. Plos Pathog. 2011;7:e1002043.

30. Murdock CC, Blanford S, Hughes GL, Rasgon JL, Thomas MB. Temperature alters Plasmodium blocking by Wolbachia. Clin Rep UK. 2014;4:3932.

31. Teo TH, Lum FM, Ghaffar K, Chan YH, Amrun SN, Tan JJL, et al. Plasmodium co-infection protects against chikungunya virus-induced pathologies. Nat Commun. 2018;9:3905.

32. Teo TH, Howland SW, Claser C, Gun SY, Poh CM, Lee WW, et al. Co-infection with chikungunya virus alters trafficking of pathogenic CD8(+) T cells into the brain and prevents Plasmodium-induced neuropathology. EMBO Mol Med. 2018;10:121-38.

33. McFarlane AJ, McSorley HJ, Davidson DJ, Fitch PM, Errington C, Mackenzie $\mathrm{KJ}$, et al. Enteric helminth-induced type I interferon signaling protects against pulmonary virus infection through interaction with the microbiota. J Allergy Clin Immunol. 2017;140:1068-78.

34. Wuerthner VP, Hua J, Hoverman JT. The benefits of coinfection: trematodes alter disease outcomes associated with virus infection. J Anim Ecol. 2017;86: 921-31.

35. Chowaniec W, Wescott RB, Congdon LL. Interaction of Nematospiroides dubius and influenza virus in mice. Exp Parasitol. 1972;32:33-44.

36. Brutus $L$, Watier $L$, Hanitrasoarnampionona V, Razanatsoarilala H, Cot M. Confirmation of the protective effect of Ascaris lumbricoides on Plasmodium falciparum infection: results of a randomized trial in Madagascar. Am J Trop Med Hyg. 2007;77:1091-5.

37. Brutus L, Watier L, Briand V, Hanitrasoamampionona V, Razanatsoarilala $H_{\text {, }}$ Cot M. Parasitic co-infections: does Ascaris lumbricoides protect against Plasmodium falciparum infection? Am J Trop Med Hyg. 2006;75:194-8.

38. Kirwan P, Jackson AL, Asaolu SO, Molloy SF, Abiona TC, Bruce MC, et al. Impact of repeated four-monthly anthelmintic treatment on Plasmodium infection in preschool children: a double-blind placebo-controlled randomized trial. Bmc Infect Dis. 2010;10:277.

39. Graham AL, Lamb TJ, Read AF, Allen JE. Malaria-filaria coinfection in mice makes malarial disease more severe unless filarial infection achieves patency. J Infect Dis. 2005;191:410-21.

40. Aliota MT, Chen CC, Dagoro H, Fuchs JF, Christensen BM. Filarial worms reduce Plasmodium infectivity in mosquitoes. Plos Negl Trop Dis. 2011;5:e963.

41. Kwan JL, Seitz AE, Fried M, Lee KL, Metenou S, Morrison R, et al. Seroepidemiology of helminths and the association with severe malaria among infants and young children in Tanzania. Plos Negl Trop Dis. 2018;12:e0006345.

42. Verma R, Kushwaha V, Pandey S, Thota JR, Vishwakarma P, Parmar N, et al. Leishmania donovani molecules recognized by sera of filaria infected host facilitate filarial infection. Parasitol Res. 2018;117:2901-12

43. Kotob MH, Gorgoglione B, Kumar G, Abdelzaher M, Saleh M, El-Matbouli M. The impact of Tetracapsuloides bryosalmonae and Myxobolus cerebralis coinfections on pathology in rainbow trout. Parasit Vectors. 2017;10:442.

44. JL DELC, Venuto Moura AP, Franca-Silva JC, DESG M, Oliveira Silva S, Norma Melo $M$, et al. Experimental mixed infection of Leishmania (Leishmania) amazonensis and Leishmania (L.) infantum in hamsters (Mesocricetus auratus). Parasitol. 2017:144:1191-202.

45. Ademola IO, Odeniran PO. Co-infection with Plasmodium berghei and Trypanosoma brucei increases severity of malaria and trypanosomiasis in mice. Acta Trop. 2016;159:29-35.

46. Soares $L$, Ellis VA, Ricklefs RE. Co-infections of haemosporidian and trypanosome parasites in a North American songbird. Parasitol. 2016;143:1930-8.

47. Lyke KE, Dicko A, Dabo A, Sangare L, Kone A, Coulibaly D, et al. Association of Schistosoma haematobium infection with protection against acute 
Plasmodium falciparum malaria in Malian children. Am J Trop Med Hyg. 2005;73:1124-30.

48. Lyke KE, Dabo A, Sangare L, Arama C, Daou M, Diarra I, et al. Effects of concomitant Schistosoma haematobium infection on the serum cytokine levels elicited by acute Plasmodium falciparum malaria infection in Malian children. Infect Immun. 2006;74:5718-24.

49. Diallo TO, Remoue F, Schacht AM, Charrier N, Dompnier JP, Pillet S, et al. Schistosomiasis co-infection in humans influences inflammatory markers in uncomplicated Plasmodium falciparum malaria. Parasite Immunol. 2004;26:365-9.

50. Amoani B, Ameyaw EO, Asante D-B, Armah FA, Prah J, Botchey CPK, et al. Effect of pre-existing Schistosoma haematobium infection on Plasmodium berghei multiplications in imprinting control region mice. Asian Pac J Trop Biomed. 2015;5:488-92.

51. Sokhna C, Le Hesran JY, Mbaye PA, Akiana J, Camara P, Diop M, et al. Increase of malaria attacks among children presenting concomitant infection by Schistosoma mansoni in Senegal. Malar J. 2004;3:43.

52. Legesse M, Erko B, Balcha F. Increased parasitaemia and delayed parasite clearance in Schistosoma mansoni and Plasmodium berghei co-infected mice. Acta Trop. 2004;91:161-6.

53. Sangweme D, Shiff C, Kumar N. Plasmodium yoelii: adverse outcome of nonlethal P. yoelii malaria during co-infection with Schistosoma mansoni in BALB/c mouse model. Exp Parasitol. 2009;122:254-9.

54. Wilson $\mathrm{S}$, Vennervald BJ, Kadzo H, Ireri E, Amaganga C, Booth $\mathrm{M}$, et al. Hepatosplenomegaly in Kenyan schoolchildren: exacerbation by concurrent chronic exposure to malaria and Schistosoma mansoni infection. Trop Med Int Health. 2007;12:1442-9.

55. Roussilhon C, Brasseur P, Agnamey P, Perignon J-L, Druilhe P. Understanding human-Plasmodium falciparum immune interactions uncovers the immunological role of worms. Plos One. 2010;5:e9309.

56. Boel M, Carrara VI, Rijken M, Proux S, Nacher M, Pimanpanarak M, et al. Complex interactions between soil-transmitted helminths and malaria in pregnant women on the Thai-Burmese border. Plos Negl Trop Dis. 2010;4:e887.

57. Courtin D, Djilali-Saiah A, Milet J, Soulard V, Gaye O, Migot-Nabias F, et al. Schistosoma haematobium infection affects Plasmodium falciparum-specific $\lg \mathrm{G}$ responses associated with protection against malaria. Parasite Immunol. 2011:33:124-31.

58. Wiria AE, Prasetyani MA, Hamid F, Wammes L, Lell B, Ariawan I, et al. Does treatment of intestinal helminth infections influence malaria? Background and methodology of a longitudinal study of clinical, parasitological and immunological parameters in Nangapanda, Flores, Indonesia (ImmunoSPIN Study). Bmc Infect Dis. 2010;10:77.

59. Florey LS, King $\mathrm{CH}$, Van Dyke MK, Muchiri EM, Mungai PL, Zimmerman PA, et al. Partnering parasites: evidence of synergism between heavy Schistosoma haematobium and Plasmodium species infections in Kenyan children. Plos Negl Trop Dis. 2012;6:e1723.

60. Wiria AE, Hamid F, Wammes L, Kaisar MM, May L, Prasetyani MA, et al. The effect of three-monthly albendazole treatment on malarial parasitemia and allergy: a household-based cluster-randomized, double-blind, placebocontrolled trial. Plos One. 2013;8:e57899.

61. Read AF, Taylor LH. The ecology of genetically diverse infections. Science. 2001;292:1099-102.

62. Bilenko N, Levy A, Dagan R, Deckelbaum RJ, El-On Y, Fraser D. Does coinfection with Giardia lamblia modulate the clinical characteristics of enteric infections in young children? Eur J Epidemiol. 2004;19:877-83.

63. Furze RC, Hussell T, Selkirk ME. Amelioration of influenza-induced pathology in mice by coinfection with Trichinella spiralis. Infect Immun. 2006;74:1924-32.

64. Rossi M, Castiglioni P, Hartley MA, Eren RO, Prevel F, Desponds C, et al. Type I interferons induced by endogenous or exogenous viral infections promote metastasis and relapse of leishmaniasis. Proc Natl Acad Sci USA. 2017;114: 4987-92.

65. Dietze KK, Dittmer U, Koudaimi DK, Schimmer S, Reitz M, Breloer M, et al. Filariae-retrovirus co-infection in mice is associated with suppressed virusspecific lgG immune response and higher viral loads. Plos Negl Trop Dis. 2016;10:e0005170.

66. Attallah AM, Abdallah SO, Albannan MS, Omran MM, Attallah AA, Farid K. Impact of hepatitis C virus/Schistosoma mansoni coinfection on the circulating levels of HCV-NS4 protein and extracellular-matrix deposition in patients with different hepatic fibrosis stages. Am J Trop Med Hyg. 2016;95:1044-50.

67. Li W, Dong H, Huang Y, Chen T, Kong X, Sun H, et al. Clonorchis sinensis coinfection could affect the disease state and treatment response of HBV patients. Plos Negl Trop Dis. 2016;10:e0004806.
68. Knowles SC, Webster BL, Garba A, Sacko M, Diaw OT, Fenwick A, et al. Epidemiological interactions between urogenital and intestinal human schistosomiasis in the context of praziquantel treatment across three west African countries. Plos Negl Trop Dis. 2015;9:e0004019.

69. Mazigo HD, Nuwaha F, Wilson S, Kinung'hi SM, Morona D, Waihenya R, et al. Epidemiology and interactions of Human Immunodeficiency Virus-1 and Schistosoma mansoni in sub-Saharan Africa. Infect Dis Poverty. 2013;2:2.

70. Chukwuanukwu RC, Ukaejiofo EO, Ele PU, Onyenekwe CC, Chukwuanukwu TO, Ifeanyichukwu MO. Evaluation of some haemostatic parameters in falciparum malaria and HIV co-infection. Br J Biomed Sci. 2016;73:168-73.

71. Hochman SE, Madaline TF, Wassmer SC, Mbale E, Choi N, Seydel KB, et al. Fatal pediatric cerebral malaria Is associated with intravascular monocytes and platelets that are increased with HIV coinfection. MBio. 2015;6:e01390-15.

72. Mooney JP, Lokken KL, Byndloss MX, George MD, Velazquez EM, Faber F, et al. Inflammation-associated alterations to the intestinal microbiota reduce colonization resistance against non-typhoidal Salmonella during concurrent malaria parasite infection. Sci Rep. 2015:5:14603.

73. Chukwuanukwu RC, Onyenekwe CC, Martinez-Pomares L, Flynn R, Singh S, Amilo Gl, et al. Modulation of the immune response to Mycobacterium tuberculosis during malaria/M. tuberculosis co-infection. Clin Exp Immunol. 2017;187:259-68.

74. Mhimbira F, Hella J, Said K, Kamwela L, Sasamalo M, Maroa T, et al. Prevalence and clinical relevance of helminth co-infections among tuberculosis patients in urban Tanzania. Plos Negl Trop D. 2017;11:e0005342.

75. Li XX, Chen JX, Wang LX, Sun J, Chen SH, Chen JH, et al. Profiling B and T cell immune responses to co-infection of Mycobacterium tuberculosis and hookworm in humans. Infect Dis Poverty. 2015;4:20.

76. Salazar-Castanon VH, Legorreta-Herrera M, Rodriguez-Sosa M. Helminth parasites alter protection against Plasmodium infection. Biomed Res Int. 2014;2014:913696

77. Adegnika AA, Kremsner PG. Epidemiology of malaria and helminth interaction: a review from 2001 to 2011. Curr Opin HIV AIDS. 2012;7:221-4.

78. Knowles SCL. The effect of helminth co-infection on malaria in mice: A meta-analysis. Int J Parasitol. 2011;41:1041-51.

79. Nacher M. Interactions between worms and malaria: Good worms or bad worms? Malaria J. 2011;10:259.

80. Hoverman JT, Hoye BJ, Johnson PT. Does timing matter? How priority effects influence the outcome of parasite interactions within hosts. Oecologia. 2013;173:1471-80

81. Diallo TOE, Remoue F, Gaayeb L, Schacht AM, Charrier N, De Clerck D, et al. Schistosomiasis coinfection in children influences acquired immune response against Plasmodium falciparum malaria antigens. Plos One. 2010;5:e12764.

82. Ulrich Y, Schmid-Hempel P. Host modulation of parasite competition in multiple infections. Proc Biol Sci. 2012;279:2982-9.

83. Davenport GC, Hittner JB, Otieno V, Karim Z, Mukundan H, Fenimore PW, et al. Reduced Parasite Burden in Children with Falciparum Malaria and Bacteremia Coinfections: Role of Mediators of Inflammation. Mediators Inflamm. 2016;2016:4286576.

84. de Rezende MC, Araujo ES, Moreira JM, Rodrigues VF, Rodrigues JL, Pereira $C A$, et al. Effect of different stages of Schistosoma mansoni infection on the parasite burden and immune response to Strongyloides venezuelensis in coinfected mice. Parasitol Res. 2015;114:4601-16.

85. Wilson S, Dunne DW. Advances in our understanding of the epidemiology of Plasmodium and schistosome infection: informing coinfection studies. Curr Opin HIV AIDS. 2012;7:225-30.

86. Alcantara-Neves NM, de SG Britto G, Veiga RV, Figueiredo CA, Fiaccone RL, da Conceicao JS, et al. Effects of helminth co-infections on atopy, asthma and cytokine production in children living in a poor urban area in Latin America. Bmc Res Notes. 2014;7:817.

87. Ezenwa VO, Jolles AE. From host immunity to pathogen invasion. the effects of helminth coinfection on the dynamics of microparasites. Integr Comp Biol. 2011;51:540-51.

88. Hoeve MA, Mylonas KJ, Fairlie-Clarke KJ, Mahajan SM, Allen JE, Graham AL. Plasmodium chabaudi limits early Nippostrongylus brasiliensis-induced pulmonary immune activation and Th2 polarization in co-infected mice. Bmc Immunol. 2009;10:60.

89. Salgame P, Yap GS, Gause WC. Effect of helminth-induced immunity on infections with microbial pathogens. Nat Immunol. 2013;14:1118-26.

90. Skapenko A, Niedobitek GU, Kalden JR, Lipsky PE, Schulze-Koops H. Generation and regulation of human Th1-biased immune responses in vivo: A critical role for IL-4 and IL-10. J Immunol. 2004;172:6427-34. 
91. Waknine-Grinberg JH, Gold D, Ohayon A, Flescher E, Heyfets A, Doenhoff $\mathrm{MJ}$, et al. Schistosoma mansoni infection reduces the incidence of murine cerebral malaria. Malaria J. 2010;9:5.

92. MacDonald AS, Patton EA, La Flamme AC, Araujo MI, Huxtable CR, Bauman $B$, et al. Impaired Th2 development and increased mortality during Schistosoma mansoni infection in the absence of CD40/CD154 interaction. J Immunol. 2002;168:4643-9.

93. Zwingenberger K, Hohmann A, de Brito MC, Ritter M. Impaired balance of interleukin-4 and interferon-gamma production in infections with Schistosoma mansoni and intestinal nematodes. Scand J Immunol. 1991;34:243-51.

94. Wang ML, Cao YM, Luo EJ, Zhang Y, Guo YJ. Pre-existing Schistosoma japonicum infection alters the immune response to Plasmodium berghei infection in C57BL/6 mice. Malaria J. 2013;12:322.

95. Wang ML, Feng YH, Pang W, Qi ZM, Zhang Y, Guo YJ, et al. Parasite densities modulate susceptibility of mice to cerebral malaria during co-infection with Schistosoma japonicum and Plasmodium berghei. Malaria J. 2014;13:116.

96. Lyke KE, Wang A, Dabo A, Arama C, Daou M, Diarra I, et al. Antigen-Specific B Memory Cell Responses to Plasmodium falciparum malaria antigens and Schistosoma haematobium antigens in co-Infected Malian children. Plos One. 2012;7:e37868.

97. Doumbo S, Tran TM, Sangala J, Li S, Doumtabe D, Kone Y, et al. Co-infection of long-term carriers of Plasmodium falciparum with Schistosoma haematobium enhances protection from febrile malaria: a prospective cohort study in Mali. PLoS Negl Trop Dis. 2014;8:e3154.

98. Naing C, Whittaker MA, Nyunt-Wai V, Reid SA, Wong SF, Mak JW, et al. Malaria and soil-transmitted intestinal helminth co-infection and its effect on anemia: a meta-analysis. Trans R Soc Trop Med Hyg. 2013;107:672-83.

99. Kolbaum J, Eschbach ML, Steeg C, Jacobs T, Fleischer B, Breloer M. Efficient control of Plasmodium yoelii infection in BALB/C and C57BL/6 mice with pre-existing Strongyloides ratti infection. Parasite Immunol. 2012;34:388-93.

100. Nitcheu J, Bonduelle O, Combadiere C, Tefit M, Seilhean D, Mazier D, Combadiere B. Perforin-dependent brain-infiltrating cytotoxic CD8+ T lymphocytes mediate experimental cerebral malaria pathogenesis. J Immunol. 2003;170:2221-8

101. Engwerda CR, Mynott TL, Sawhney S, De Souza JB, Bickle QD, Kaye PM. Locally up-regulated lymphotoxin alpha, not systemic tumor necrosis factor alpha, is the principle mediator of murine cerebral malaria. J Exp Med. 2002; 195:1371-7.

102. Specht S, Ruiz DF, Dubben B, Deininger S, Hoerauf A. Filaria-induced IL-10 suppresses murine cerebral malaria. Microbes Infect. 2010;12:635-42.

103. Boef AG, May L, van Bodegom D, van Lieshout L, Verweij JJ, Maier AB, et al. Parasitic infections and immune function: effect of helminth infections in a malaria endemic area. Immunobiol. 2013:218:706-11.

104. Turner JD, Meurs L, Dool P, Bourke CD, Mbow M, Dieye TN, et al. Schistosome infection is associated with enhanced whole-blood IL-10 secretion in response to cercarial excretory/secretory products. Parasite Immunol. 2013;35:147-56.

105. Noland GS, Urban JF Jr, Fried B, Kumar N. Counter-regulatory anti-parasite cytokine responses during concurrent Plasmodium yoelii and intestinal helminth infections in mice. Exp Parasitol. 2008;119:272-8.

106. Dong Y, Manfredini F, Dimopoulos G. Implication of the mosquito midgut microbiota in the defense against malaria parasites. Plos Pathog. 2009;5:e1000423.

107. Craig JM, Scott AL. Antecedent Nippostrongylus infection alters the lung immune response to Plasmodium berghei. Parasite Immunol. 2017;39:10.

108. Renia L, Potter SM. Co-infection of malaria with HIV: an immunological perspective. Parasite Immunol. 2006;28:589-95.

109. Karp CL, Auwaerter PG. Coinfection with HIV and tropical infectious diseases. II. Helminthic, fungal, bacterial, and viral pathogens. Clin Infect Dis. 2007:45:1214-20

110. Santoro F, Prata A, Castro CN, Capron A. Circulating antigens, immune complexes and C3d levels in human schistosomiasis: relationship with Schistosoma mansoni egg output. Clin Exp Immunol. 1980;42:219-25.

111. Dempsey PW, Allison MED, Akkaraju S, Goodnow CC, Fearon DT. C3d of complement as a molecular adjuvant: Bridging innate and acquired immunity. Science. 1996;271:348-50.

112. Chu KB, Kim SS, Lee SH, Lee HS, Joo KH, Lee JH, et al. Enhanced protection against Clonorchis sinensis induced by co-infection with Trichinella spiralis in rats. Parasite Immunol. 2014;36:522-30.

113. Chen Y, Huang B, Huang S, Yu X, Li Y, Song W, et al. Coinfection with Clonorchis sinensis modulates murine host response against Trichinella spiralis infection. Parasitol Res. 2013;112:3167-79.
114. Budischak SA, Wiria AE, Hamid F, Wammes LJ, Kaisar MMM, van Lieshout L, et al. Competing for blood: the ecology of parasite resource competition in human malaria-helminth co-infections. Ecol Lett. 2018;21:536-45.

115. Rauque CA, Semenas L. Interactions among four parasite species in an amphipod population from Patagonia. J Helminthol. 2013;87:97-101.

116. Mideo N. Parasite adaptations to within-host competition. Trends Parasitol. 2009:25:261-8

117. Wale N, Sim DG, Read AF. A nutrient mediates intraspecific competition between rodent malaria parasites in vivo. Proc Biol Sci. 2017;284:20171067.

118. Deans AM, Lyke KE, Thera MA, Plowe CV, Kone A, Doumbo OK, et al. Low multiplication rates of African Plasmodium falciparum isolates and lack of association of multiplication rate and red blood cell selectivity with malaria virulence. Am J Trop Med Hyg. 2006;74:554-63.

119. Xong HV, Vanhamme L, Chamekh M, Chimfwembe CE, Van Den Abbeele J, Pays A, et al. A VSG expression site-associated gene confers resistance to human serum in Trypanosoma rhodesiense. Cell. 1998;95:839-46.

120. Naus CWA, Jones FM, Satti MZ, Joseph S, Riley EM, Kimani G, et al. Serological responses among individuals in areas where both schistosomiasis and malaria are endemic: Cross-reactivity between Schistosoma mansoni and Plasmodium falciparum. J Infect Dis. 2003;187:1272-82.

121. Tunholi VM, Monteiro CO, Cristina da Silva L, Dolinski Cde M, José dos Santos MA, Rodrigues Mde L, et al. Physiological alterations in Bradybaena similaris (Stylommatophora: Bradybaenidae) induced by the entomopathogenic nematode Heterorhabditis indica (Rhabditida: Heterorhabditidae) strain LPP1. Exp Parasitol. 2014;139:12-8.

122. Tarasco E, Clausi M, Rappazzo G, Panzavolta T, Curto G, Sorino R, et al. Biodiversity of entomopathogenic nematodes in Italy. J Helminthol. 2015;89:359-66.

123. Samish M, Glazer I. Entomopathogenic nematodes for the biocontrol of ticks. Trends Parasitol. 2001;17:368-71.

124. Desenclos JC. Transmission parameters of vector-borne infections. Med Mal Infect. 2011:41:588-93.

125. Tunholi-Alves VM, Tunholi VM, Pinheiro J, Thiengo SC. Effects of infection by larvae of Angiostrongylus cantonensis (Nematoda, Metastrongylidae) on the metabolism of the experimental intermediate host Biomphalaria glabrata. Exp Parasitol. 2012;131:143-7.

126. dos Santos Bonfim TC, Maldonado A Jr, Tunholi VM, Tunholi-Alves VM, Faro MJ, Mota EM, et al. Biochemical and histopathological alterations in Biomphalaria glabrata due to co-infection by Angiostrongylus cantonensis and Echinostoma paraensei. J Invertebr Pathol. 2014;115:80-5.

127. Jeffries $\mathrm{CL}$, Walker T. Wolbachia biocontrol strategies for arboviral diseases and the potential influence of resident Wolbachia strains in mosquitoes. Curr Trop Med Rep. 2016:3:20-5.

128. Slatko BE, Luck AN, Dobson SL, Foster JM. Wolbachia endosymbionts and human disease control. Mol Biochem Parasit. 2014;195:88-95.

129. Pan X, Pike A, Joshi D, Bian G, McFadden MJ, Lu P, et al. The bacterium Wolbachia exploits host innate immunity to establish a symbiotic relationship with the dengue vector mosquito Aedes aegypti. ISME J. 2018;12:277-88.

130. Kim W, Koo H, Richman AM, Seeley D, Vizioli J, Klocko AD, et al. Ectopic expression of a cecropin transgene in the human malaria vector mosquito Anopheles gambiae (Diptera: Culicidae): Effects on susceptibility to Plasmodium. J Med Entomol. 2004;41:447-55.

131. McMeniman CJ, Lane RV, Cass BN, Fong AWC, Sidhu M, Wang Y-F, et al. Stable introduction of a life-shortening Wolbachia infection into the mosquito Aedes aegypti. Science. 2009;323:141-4.

132. Imai N, Rujeni N, Nausch N, Bourke CD, Appleby LJ, Cowan G, et al. Exposure, infection, systemic cytokine levels and antibody responses in young children concurrently exposed to schistosomiasis and malaria. Parasitol. 2011;138:1519-33.

133. Karaborklu S, Azizoglu U, Azizoglu ZB. Recombinant entomopathogenic agents: a review of biotechnological approaches to pest insect control. World J Microb Biot. 2017;34:14.

134. Fairlie-Clarke KJ, Allen JE, Read AF, Graham AL. Quantifying variation in the potential for antibody-mediated apparent competition among nine genotypes of the rodent malaria parasite Plasmodium chabaudi. Infect Genet Evol. 2013;20:270-5

135. Efunshile AM, Olawale T, Stensvold CR, Kurtzhals JA, Konig B. Epidemiological study of the association between malaria and helminth infections in Nigeria. Am J Trop Med Hyg. 2015;92:578-82.

136. Mustafa MS, Rastogi V, Gupta RK, Jain S, Singh PMP, Gupta A. Wolbachia: The selfish Trojan Horse in dengue control. Med J Armed Forces India. 2016; 72:373-6. 